\title{
Twinkle, twinkle little stone: an artifact improves the ultrasound performance!
}

\author{
Mirela Liana Gliga ${ }^{1,2}$, Cristian Nicolae Chirila ${ }^{3}$, Daniela Maria Podeanu ${ }^{4}$, Torok Imola ${ }^{1}$, \\ Sanda Lucia Voicu ${ }^{5}$, Mihail Gheorghe Gliga ${ }^{6}$, Paula Maria Gliga ${ }^{3}$
}

\begin{abstract}
${ }^{1}$ Department of Internal Medicine, University of Medicine and Pharmacy Targu Mures, ${ }^{2}$ Diaverum Dialysis Centre, Targu Mures, ${ }^{3}$ University of Medicine and Pharmacy Targu Mures, ${ }^{4}$ Department of Radiology, University of Medicine and Pharmacy Targu Mures, ${ }^{5}$ Department of Pediatrics, University of Medicine and Pharmacy Targu Mures, ${ }^{6}$ Department of Physiology, University of Medicine and Pharmacy Targu Mures, Romania
\end{abstract}

\begin{abstract}
Aims: Ultrasound is a noninvasive method used for the diagnosis of urinary lithiasis. When the length of the stone is less than $5 \mathrm{~mm}$, its detection may be difficult. The twinkling artifact (TwA) is an intense alternating color signal behind calcifications and stones in different organs. The aim of this paper was to evaluate the importance of the TwA in detecting kidney stones less than $5 \mathrm{~mm}$ in length. Material and methods: We examined 230 patients with lumbar pain or a history of kidney stones. We excluded patients with stones larger than $5 \mathrm{~mm}$. 174 patients corresponded to the inclusion criteria. We performed color Doppler ultrasound and we noticed the presence of the twinkling artifact. The gold standard for the diagnosis was either computed tomography (CT) scan, intravenous urography (IVU) or the spontaneous elimination of the stones. Results: We found renal stones in 123 patients. The twinkling artifact was present in 113 cases and absent in the rest of 10 patients. In two patients the artifact was present but the stones were not confirmed by CT. The twinkling artifact had sensitivity, specificity, positive predictive value and negative predictive value of $99.12 \%, 90.91 \%, 99.12 \%$, and $90.91 \%$ respectively. Conclusions: The twinkling artifact is a very useful color Doppler ultrasound tool for the detection of small urinary stones. We suggest the routine use of color Doppler in all suspicious cases in order to avoid unnecessary irradiating and expensive radiological methods.
\end{abstract}

Keywords: twinkling artifact; color Doppler ultrasound; renal stones

\section{Introduction}

Kidney stones can have many clinical manifestations, depending on their location, length, surface, and number. These manifestations can vary from typical renal colic with acute lumbar pain to asymptomatic cases with unexplained recurrent urinary infections [1]. Therefore, their identification is mandatory in order to confirm the diagnosis and to apply the proper treatment. Ultrasound (US) is an inexpensive, non-invasive, radiation-free method used by nephrologists, urologists, and other medical specialists on a daily basis, but it can have many false negative

Received 29.01.2017 Accepted 15.05.2017

Med Ultrason

2017, Vol. 19, No 3, 272-275

Corresponding author: Cristian Chirila

6A Borzesti street

Targu Mures 540075, Romania

Phone: +40721134660

E-mail: cristi_nic94@yahoo.com or positive results due to the anatomic and US characteristics of the intrarenal structures [2]. As the echogenicity of a stone is higher than the surrounding structures, such as caliceal walls, arterial or vein walls and adipose tissue from the renal sinus, the stone is easy to visualize if it is longer than $5 \mathrm{~mm}$.. But when its dimensions are less than $5 \mathrm{~mm}$ and there is an increase in the echogenicity of the central area of the kidney - frequently seen in older people - the detection of the stone can be difficult.

The twinkling artifact (TwA), also called "the color comet tail artifact", is a complex phenomenon, an intense color signal produced by calcifications in different organs $[3,4]$. The signal is persistent even if the pulse repetition frequency (PRF) is adjusted to higher levels. In pulsed wave (PW) mode, no flow can be detected; only linear lines with a disturbing audio-signal.

The aim of this paper is to evaluate the importance of the TwA in detecting small urinary stones less than 5 $\mathrm{mm}$ in length. 


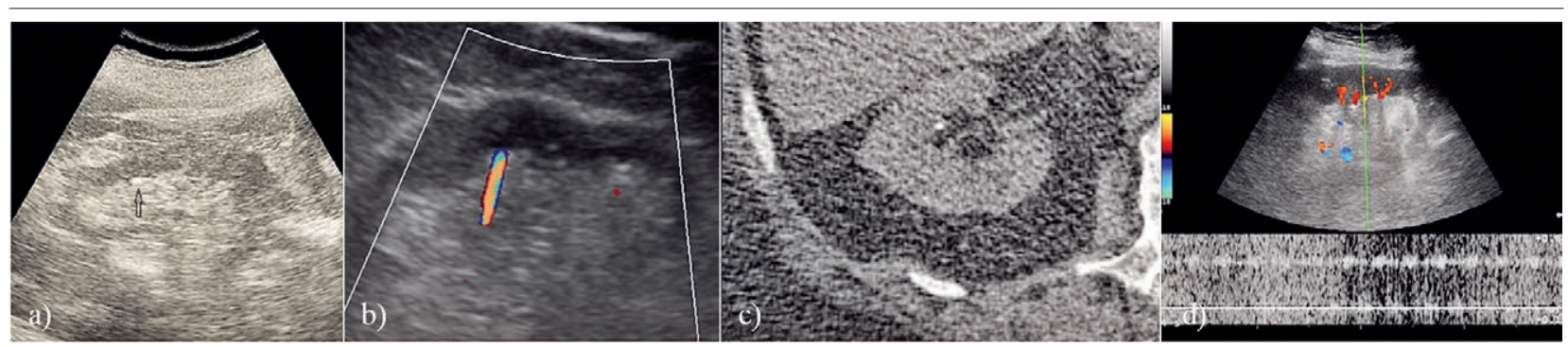

Fig 1. a) Right kidney: small hyperechogenic image in the renal sinus (arrow), without posterior acoustic shadow; b) Color Doppler at the same patient with the visualization of the TwA; c) CT scan image in same patient that confirmed the lithiasis; d) Pulsed Wave examination with the sample volume localized in the TwA: noisy signal and characteristic parallel lines.

\section{Material and methods}

We prospectively examined by US 230 patients admitted to the emergency room of the Nephrology Department from Mures County Hospital during a period of three months, between March and May 2014. All patients signed an informed consent and the local Ethics Committee approved the protocol of the study. We used the same range settings of the US device for all the cases and we depicted the appearance of the TwA over the central echogenic area within the kidney using US color Doppler.

Inclusion criteria were: uni/bilateral lumbar pain (colicative or not) or history of kidney stones. The patients with stones larger than $5 \mathrm{~mm}$ were excluded. After grey-scale US examination we selected 174 patients. Data about history of urinary lithiasis, presence of crystals in the urine sediment, previous CT-scan or intra-venous urography (IVU) conclusive for lithiasis were collected.

\section{US examination}

US examinations were performed at the Ultrasound Unit of the Nephrology Department, Mures County Hospital, between March and May 2014. All examinations were performed by a single examiner with over 10 years of experience in renal US, blinded to the patients' history. An Esaote Mylab 50 machine with a 3.5 $\mathrm{MHz}$ convex array abdominal transducer was used. The technical parameters used were: emission frequency 3.5 $\mathrm{MHz}$, color frequency $2.5 \mathrm{MHz}$, Gain 60-70, Filter 5, TIS 1.2, and MI 1.5. Standard US examination of the kidneys was performed following precise steps. First, both kidneys and urinary bladder were investigated by grey scale US followed by a complete abdominal and pelvic examination. The morphologies of the parenchyma and of the central echogenic complex were recorded for both kidneys. Then, in cases with US suspicion of intrarenal stones, color Doppler investigation was realized. Whenever a twinkling sign was present, a pulsed Doppler was performed to exclude arterial or venous flow. If the sam- ple volume placed on the color mosaic displayed parallel vertical bands on both sides of the baseline with a disturbing noise the aspect was considered to be TwA. Also, the PRF was set over $60 \mathrm{~cm} / \mathrm{s}$ in order to rule out the arterial signal. In these settings, if the TwA remained visible, the aspect was interpreted as artifact (fig 1).

After US examination the patients were supplementary investigated (urine sediment exam, CT scan or IVU), according to the clinician indications for each patient. These methods and/or the spontaneous elimination of the calculi were considered to be methods for confirmations of the lithiasis.

\section{Statistic analysis}

Statistical analysis was performed with MedCalc software and the following parameters were calculated for $95 \%$ confidence interval: sensitivity, specificity, positive predictive value (PPV) and negative predictive value (NPV).

\section{Results}

The presence of renal stones was confirmed in 123 out of 174 patients (37 patients using CT-scans, 20 patients using IVU, and 66 patients had history of lithiasis combined with the presence of urine crystals and spontaneous elimination of the calculi). The TwA was present in 113 of these patients. In 10 patients with confirmed renal stones the TwA was not detected and in 2 patients the TwA was present in the absence of the stones (stones not found at CT examination).

The sensitivity, specificity, PPV and NPV of the TwA in detecting renal stones less than $5 \mathrm{~mm}$ was $99.12 \%$, $90.91 \%, 99.12 \%$ and $90.91 \%$ respectively.

\section{Discussions}

Previous observations from the literature stated that the appearance of the TwA is dependent on the machine settings, the biochemical components of the stones and the surface of the calcification. Few studies have been 
carried out regarding the dimensions of the kidney stones and they included a small number of patients [5]. The experience of the examiner can be also an issue. By being aware of this artifact we can improve the detection of lithiasis, especially in the kidney.

The TwA is a complex phenomenon. First described in 1996 by Rahmouni et al, it consists of an intense alternating color signal between red and blue behind some structures [5]. Two theories have been proposed to explain the TwA. The first one was offered by Rahmouni et al, who suggested that this artifact is generated by a strongly reflecting medium with a rough interface. They explained that, when an incidental ultrasound beam is reflected by a flat interface, the acoustic waves are reflected by the interface at the same time and so it results in the production of short-wave sound signals. When the incidental beam is reflected on a rough interface, the acoustic wave is split into a complex beam pattern caused by multiple reflections in the medium, resulting in prolonged pulse duration of the transmitted sound signal and the Doppler units interpret this result as movement and thus assign it different colors. The authors observed that parenchymal calcifications and bladder or gallbladder calculi were sources of a rapidly changing mixture of red and blue. They used spectral Doppler US to confirm the presence of the calcifications and the noise could be heard in the audio mode. Additionally, Hirsh et al performed several experiments, which showed the TwA behind granular structures such as sodium chloride, iron filings, emery paper and ground chalk [6]. The second theory was offered by Kamaya et al, who stated that the TwA is caused by a narrow band of intrinsic sonographic machine noise, referred to as phase or clock jitter, which may be generated by slight random time fluctuations in the path lengths of transmitted and reflected acoustic waves [7].

However, the most accepted theory nowadays is that the presence of a narrow-band noise due to fluctuations in the circuits of Doppler US equipment is the underlying cause of this sign. It is mainly observed on rough, hyperechoic, irregular surfaces with multiple cracks which cause a strong reflection of the incident US waves and multiple internal reflections which widen the spectrum. The appearance of the TwA is correlated with the roughness of the calculi - the greater the surface roughness, the greater the artifact [8]. This can explain why in 10 cases we did not find the TwA due to smooth, regular interface of the stones surface.

In the human body, the TwA appears behind calcifications in different organs and has an important clinical relevance in many cases. It can be found in other diseases beside urinary lithiasis such as encrusted ureteral stents, choledocholithiasis, coils used for embolization in the cerebrovascular system, adenomyomatosis of the gallbladder, bile duct hamartomas, ductal and parenchymal calcifications in chronic pancreatitis, vascular calcification, surgical clips, balloon of the bladder catheter or foreign bodies. It is also dependent on the machine parameters and the US emission frequency; therefore, we used the same settings in all cases [9]. One of the machine settings which may influence the TwA is the location of the focal zone: if the focal zone is located below a rough reflecting surface, the TwA becomes more obvious comparing with the position of the focal zone above this surface. Other machine settings that influence the appearance of the TwA are color-write priority, gray-scale gain, color Doppler frequency and color Doppler gain. Colorwrite priority determines whether a pixel is depicted as color or as gray scale. Increasing the color-write priority causes more color information to be displayed and thus enhances the TwA. However, increasing gray-scale gain will reduce the size of the TwA. Decreasing the color Doppler frequency and increasing color Doppler gain are expected to increase the appearance of the TwA.

Only a few researchers have evaluated the use of the TwA for the detection of nephrolithiasis. However, there is evidence that suggests this artifact may substantially improve the ability of physicians to detect nephrolithiasis with US. In the in vitro study of Shabana et al, the TwA associated with renal calculi demonstrated increased contrast-to-noise ratio when compared with gray-scale posterior acoustic shadowing [10]. Other authors have suggested that this artifact should noticeably increase the detection of renal calculi when compared with traditional gray-scale US. It is sometimes difficult to detect stones with gray-scale imaging because of the obscuring effects of bowel gas. However, the TwA facilitates stone detection and its presence strongly supports a diagnosis of ureteral stone. TwA can be regarded as a significant marker for urolithiasis [11] and a major diagnosing sonographic finding in this context [12]. Even more, TwA based colorDoppler US is preferable for the sensitive detection of very small nephrolithiasis $[13,14]$. Park et al, in 318 patients, reported sensitivity and specificity rates for TwA of $98 \%$ and $100 \%$ respectively; meanwhile Korkmaz et al reported only $93 \%$ sensibility and Yavuz et al found a PPV of $88,3 \%$ for TwA $[1,11,13]$. Our results were comparable with a higher PPV.

The biochemical composition of the stones may influence the TwA. We had 4 cases with urate stones without evidence of the TwA and previous observations showed indeed no color signals over the stones although they were larger than $1 \mathrm{~cm}$. Kim et al described pitfalls in gallbladder adenomyomatosis without cholesterol deposits in which the TwA could not be visualized. Moreover, 
Chelfouh et al noticed the interrelation between the stone composition and the presence of the TwA: stones composed of calcium oxalate or calcium phosphate produced TwA, whereas most of those composed predominantly of calcium oxalate monohydrate or urate did not [14-16].

Renal artery calcification should be considered in the differential diagnosis, especially in patients with longstanding diabetes, hypertension, or other systemic diseases associated with atherosclerotic vascular disease. Real-time scanning can help differentiate arterial calcifications from renal calculi because arterial calcifications are pulsatile. However, the twinkling sign may also develop from calcifications of renal tumors, renal cysts, and renal parenchyma. These calcifications can usually be differentiated from renal stones based on their location on real-time scanning and the patient's history.

Although TwA seems to be useful in detecting small kidney stones, there are some limitations in our study. First, we had no indications about the biochemical composition of the stones. Further, we did not calculate an inter- and intraobserver agreement in order to evaluate the subjectivism of the method. The radiologist who performed the CT scans was aware of the diagnosis and therefore he could have been influenced by it. It is also possible that some calculi could have been missed on the CT scan because of the variable thickness of the slices. Other limitations of our research were the small number of patients and the non-unitary gold standard method for the diagnosis confirmation. Further studies are required and objective criteria for image interpretation must be established [16].

\section{Conclusions}

Kidney stones can be difficult to detect in some patients; therefore, we need a better way to visualize them. The TwA is a very useful US color Doppler tool for the detection of small urinary stones. Being aware of it, an experimented examiner can raise the specificity of the detection and consequently the quality of the diagnosis and treatment. Understanding the TwA may result in the better use of its clinical appearance. Color Doppler must trace specified characteristics in order to be reliable for interpretation. We propose the routine use of color Doppler in all suspicious cases as a cost-effective method that can avoid unnecessary irradiating and expensive radiological methods.

\section{References}

1. Park SJ, Yi BH, Lee HK, Kim YH, Kim GJ, Kim HC. Evaluation of patients with suspected ureteral calculi using sonography as an initial diagnostic tool: how can we improve diagnostic accuracy? J Ultrasound Med 2008;27:14411450 .

2. Ahmad SK, Abdallah MM. The diagnostic value of the twinkle sign in color Doppler imaging of urinary stones. The Egyptian Journal of Radiology and Nuclear Medicine 2014;45:569-574.

3. Ozan E, Atac GK, Gundogdu S. Twinkling artifact on color Doppler ultrasound: an advantage or a pitfall? J Med Ultrason 2016;43:361-371.

4. Bennett JM, Estrada JC, Shoemaker MB, Pretorius M. Twinkling artifact associated with guidewire placement. Anesth Analg 2015;121:69-71.

5. Rahmouni A, Bargoin R, Herment A, Bargoin N, Vasile N. Color Doppler twinkling artifact in hyperechoic regions. Radiology 1996;199:269-271.

6. Hirsch SM, Palavecino BT, León RL. Color Doppler twinkling artifact: a misunderstood and useful sign. Rev Chil Radiol 2011;17:82-84.

7. Kamaya A, Tuthill T, Rubin JM. Twinkling artifact on color Doppler sonography: dependence on machine parameters and underlying cause. AJR Am J Roentgenol 2003;180:215-222.

8. Jamzad A, Setarehdan SK. A novel approach for quantification and analysis of the color Doppler twinkling artifact with application in noninvasive surface roughness characterization: an in vitro phantom study. J Ultrasound Med $2014 ; 33: 597-610$.

9. Wang M, Li J, Xiao J, Shi D, Zhang K. Systematic analysis of factors related to display of the twinkling artifact by a phantom: an optimized investigation. J Ultrasound Med 2011;30:1449-1457.

10. Shabana W, Bude RO, Rubin JM. Comparison between color Doppler twinkling artifact and acoustic shadowing for renal calculus detection: an in vitro study. Ultrasound Med Biol 2009;35:339-350.

11. Korkmaz M, Aras B, Sanal B, et al. Investigating the clinical significance of twinkling artifacts in patients with urolithiasis smaller than 5 mm. Jpn J Radiol 2014;32:482-486.

12. Kim HC, Yang DM, Jin W, Ryu JK, Shin HC. Color Doppler twinkling artifacts in various conditions during abdominal and pelvic sonography. J Ultrasound Med 2010;29:621632.

13. Yavuz A, Ceken K, Alimoglu E, Kabaalioglu A. The reliability of color Doppler "twinkling" artifact for diagnosing milimetrical nephrolitiasis: comparison between B-mode US and CT scanning results. J Med Ultrason 2015;42:215222.

14. Chelfouh N, Grenier N, Higueret D, et al. Characterization of urinary calculi: in vitro study of "twinkling artifact" revealed by color-flow sonography. AJR Am J Roentgenol 1998;171:1055-1060.

15. Alan C, Koçoğlu H, Kosar S, Karatag O, Ersay AR, Erhan A. Role of twinkling artifact in characterization of urinary calculi. Actas Urol Esp 2011;35:396-402.

16. Mitterberger N, Aigner F, Pallwein L, et al. Sonographic detection of renal and ureteral stones. Value of the twinkling sign. Int Braz J Urol 2009;35:532-541. 